Article

\title{
The Significance of New Records of Benthic Red Algae (Rhodophyta) for Hainan Island (and China) between 1990 and 2016
}

\author{
Tamara V. Titlyanova ${ }^{1}$, Eduard A. Titlyanov ${ }^{1, *}$, Xiubao Li $^{2}$, Inka Bartsch ${ }^{3}$ and Bangmei Xia ${ }^{4}$ \\ 1 National Scientific Centre of Marine Biology, Far Eastern Branch of the Russian Academy of Sciences, \\ Palchevskogo 17, Vladivostok 690041, Russia; titlyanova@inbox.ru \\ 2 Key Laboratory of Tropical Marine Bio-Resources and Ecology, South China Sea Institute of Oceanology, \\ Chinese Academy of Sciences, Guangzhou 510301, China; lixiubao@scsio.ac.cn \\ 3 Alfred-Wegener-Institute Helmholtz-Centre for Polar and Marine Research, Am Handelshafen 12, \\ 27570 Bremerhaven, Germany; inka.bartsch@awi.de \\ 4 Institute of Oceanology, Chinese Academy of Sciences, 7 Nanhai Road, Qingdao 266071, China; \\ xsun@qdio.ac.cn \\ * Correspondence: etitlyanov@mail.ru
}

Academic Editor: Rupert Ormond

Received: 3 March 2017; Accepted: 25 May 2017; Published: 31 May 2017

\begin{abstract}
We present an annotated list of new finds of red algae from Hainan Island, Southern China, including those found in 1990 and 1992 during the German-Chinese expeditions to Hainan Island and in 2008-2016 by Titlyanova, Titlyanov, and Li. Between 1990 and 1992, a total of 64 taxa of red algae were newly recorded for Hainan Island. Of these 15 species were new records for China. During the period 2008-2016, a further 54 taxa were newly recorded for Hainan Island, of which 20 were new records for China. The full list of new taxa includes taxonomic forms, dates, and locales, together with known biogeographical distributions. During both periods, the apparent enrichment of red algal marine flora has occurred in a similar way-mainly at the expense of epiphytes with filamentous, thin-filamentous, and finely branched forms. We believe that the changes in the flora of Hainan Island have been influenced by both anthropogenic and natural factors including in particular exploitation of herbivores, nutrient pollution, and coral bleaching.
\end{abstract}

Keywords: new records; Rhodophyta; Hainan Island; China

\section{Introduction}

Hainan Island is located at the subtropical northern periphery of the Indo-Pacific in the South China Sea $\left(18^{\circ} 10^{\prime}-20^{\circ} 9^{\prime} \mathrm{N}, 108^{\circ} 37^{\prime}-111^{\circ} 1^{\prime}\right.$ E) (Figure 1). The island has an area of about $33,920 \mathrm{~km}^{2}$ and a coastline of more than 1000 miles. The annual mean sea surface temperature (SST) is $26^{\circ} \mathrm{C}$ with an average seasonal range of $12.1^{\circ} \mathrm{C}$ [1]. The annual SST maximum $\left(30.8^{\circ} \mathrm{C}\right)$ and minimum $\left(18.7^{\circ} \mathrm{C}\right)$ commonly occur in July and January, respectively. Mean sea surface salinity (SSS) in the South China Sea fluctuates between 33.3 and 34.0 practical salinity units (PSU). The mean tidal range is mostly less than $1.5 \mathrm{~m}[2,3]$. Shallow areas along Hainan Island coastline are occupied by coral reefs. Seagrass beds and mangroves are also present, but to a lesser extent [4]. The species diversity on the coral reefs appears to have been declining, as does that of the seagrass beds, mainly because of increasing tourism, aquaculture, sediment loads, pollutions from land, and the coastal engineering associated with development [4-11]. The marine algal flora of Hainan Island has been studied by a number of phycologists over the period from 1933 to 2016. The first sampling of marine seaweeds on the island was performed by mainly Chinese algologists [12], from 1933 to the 1980s, at sites covering all coasts of the island (Figure 1). 


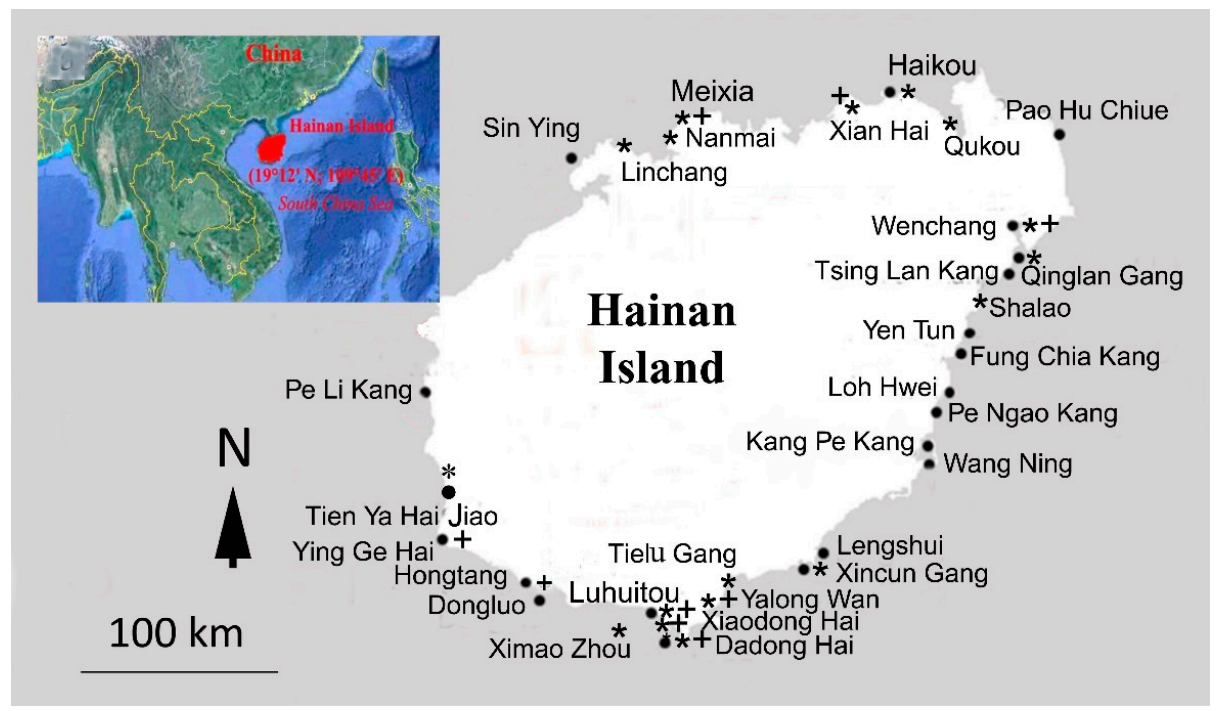

Figure 1. Algal collection sites at Hainan Island: $(\bullet)$-collection sites of Tseng and coworkers in the 1930s-1980s (old spellings of sites names); (*)—collection sites of two German-Chinese expeditions during October-December 1990 and March-April 1992; (+)—collection sites of Titlyanova, Titlyanov, and Li between 2008 and 2016.

This collection has been deposited in the herbarium of the Institute of Oceanology of the Chinese Academy of Sciences [12]. All records based on these collections were considered in our previous papers [13-18]. In this early collection (EC), a total of 310 macroalgal taxa and their forms were found, of which 178 species (57\%) were Rhodophyta (Rh), 64 (21\%) Phaeophyceae (Ph), and 68 (22\%) Chlorophyta (Ch). The next major algal sampling on Hainan was conducted in 1990 and 1992 during the German-Chinese expeditions (IC) to Hainan Island (Figure 1). The results of these expeditions were partially published [13,16-18], with 203 taxa collected, including 105 red (52\%), 32 brown (16\%), and 66 green (32\%) algal taxa. A third sampling was performed during 2008-2016 (LC) (Figure 1). Some of the results of this algal inventory have also been published [13-15,17,19,20], with 301 species being recorded including 163 (54\%) Rhodophyta, 50 (17\%) Phaeophyceae, and 88 (29\%) Chlorophyta. This further paper presents an annotated list of new findings of red algae for Hainan Island made during the course of this study. On the basis of this new list with data in the literature, decadal changes in the benthic flora of the island between the 1930s and 2000s are analyzed.

\section{Materials and Methods}

\subsection{Study Sites and Times of Sampling in 1990 and 1992}

IC algal sampling was conducted during the German-Chinese expeditions in October-December 1990 and in March-April 1992. During these expeditions, algal samples were processed as herbarium specimens by Xia and Bartsch with colleagues. New findings of red algae were documented from the following sites: Haikou and Qukou on the North coast, Qingnan Gang and Shalao on the East coast, Tielu Gang, Yalong Wan, Dadong Hai, Luhuitou, Xiaodong Hai, Ximao Zhou, and Tian Ya Hai Jiao on the South coast, and Linchang, Meixia, and Nanmai on the North-West coast (Figure 1).

At Linchang, Luhuitou, Dadong Hai, Xiaodong Hai, Yalong Wan, and Tian Ya Hai Jiao, the intertidal/infralittoral reef flat was characterized by mobile blocks of dead corals, typically on a rocky substratum interspersed with sandy areas. Poorly developed mangrove areas with extensive mudflats/seagrass fields were present at Qukou and Qingnan Gang. In Haikou, samples were collected at localities with variable salinity. The Eucheuma farm in Shalao was characterized by a sandy bay with an offshore reef flat composed of mostly dead corals. The offshore Ximao Zhou Islet was the only place where the amount of living corals increased with depths in the shallow subtidal zones and where the 
reef was generally species-rich. At Meixia, there were extensive stony intertidal areas with an offshore reef flat mostly comprised of dead corals. At Nanmai, there was an extensive reef flat with many living corals on the top of or between dead corals.

\subsection{Study Sites and Times of Sampling during 2008-2016}

Samplings of benthic macroalgae were carried out in October 2008 at Luhuitou, Dadong Hai, and Xiaodong Hai, in April 2009 at Luhuitou, in December 2010 at Luhuitou, in February-April 2012 at Luhuitou, Meixia, Wenchang, Xian Hai, and Yalong Wan, in April 2014 at Luhuitou, Xiaodong Hai, and Ying Ge Hai, and in March-April 2015 and 2016 at Luhuitou and Xiaodong Hai (Figure 1). In 2008-2016 Luhuitou (Figure 2), Xiaodong Hai (Figure 3) and Dadong Hai (Figure 4) were characterized by damaged coral reefs, estimated cover of live corals being approximately $25 \%, 10 \%$, and $3-5 \%$, respectively; seaweeds were collected in the intertidal and upper subtidal zones.

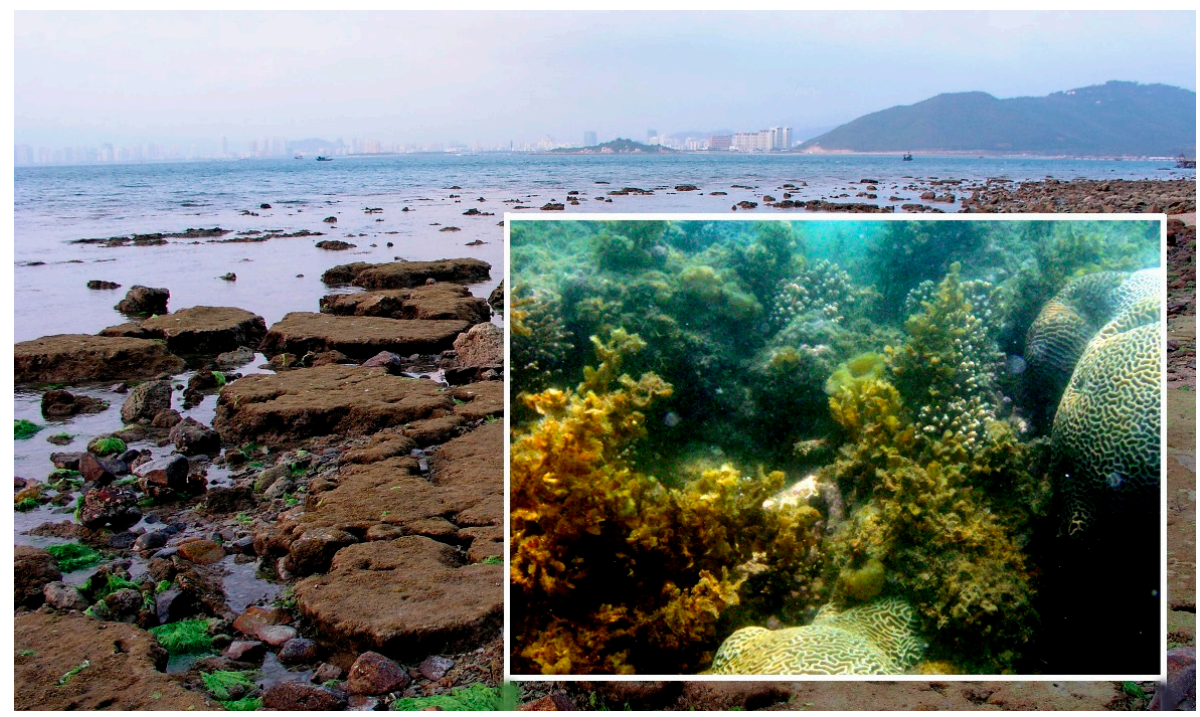

Figure 2. Sanya Bay, Luhuitou Peninsula, the intertidal zone at low tide. Inset: The uppersubtidal zone, March 2012.

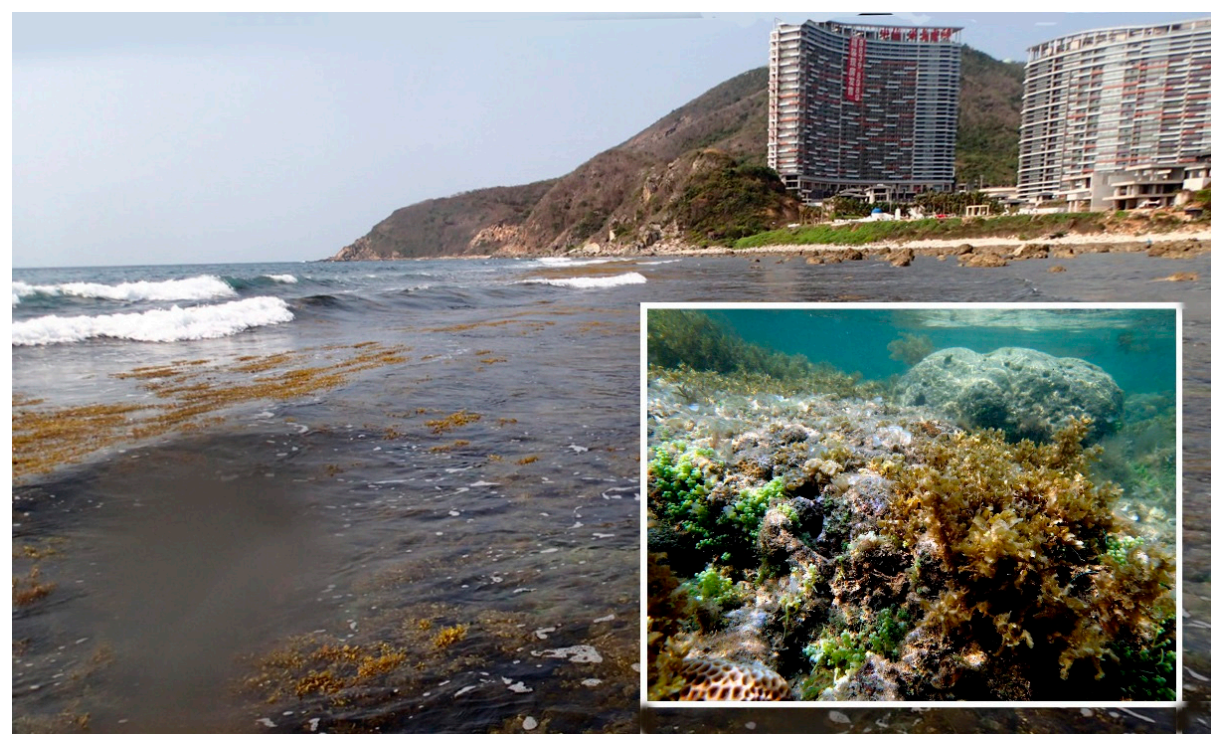

Figure 3. Xiaodong Hai, Sargassum spp. belt along the coast. Inset: The upper subtidal zone. March 2015. 


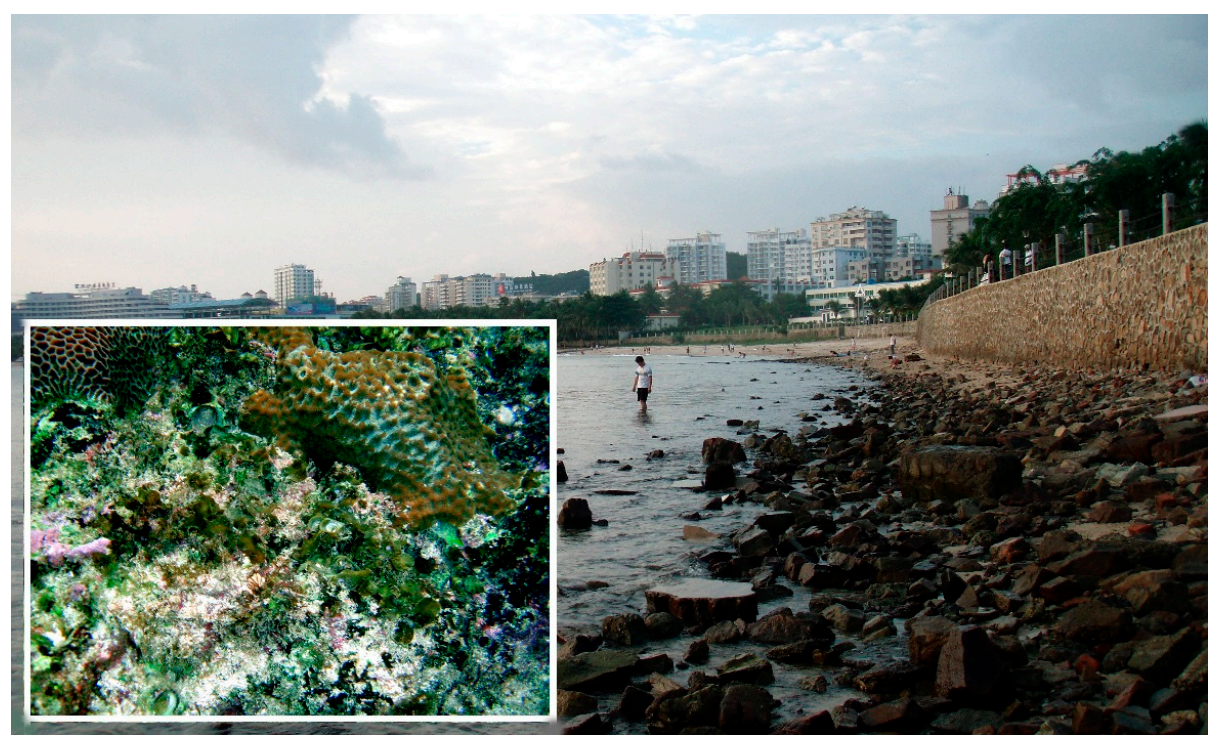

Figure 4. Dadong Hai at low tide, October 2008. Inset: Turf algae among live corals.

The locality Yalong Wan (Xipai, a small rocky islet) was characterized by a sandy bottom with blocks of dead corals, stones, and boulders, and a rocky bottom near the islet; seaweeds were sampled in the intertidal and upper subtidal zones. At the locality Wenchang (Figure 5), algae were collected from the intertidal zone on a damaged coral reef. In Xian Hai, seaweeds were collected from beach cast and fishery nets, which were placed on the coral reef, poor in live corals.

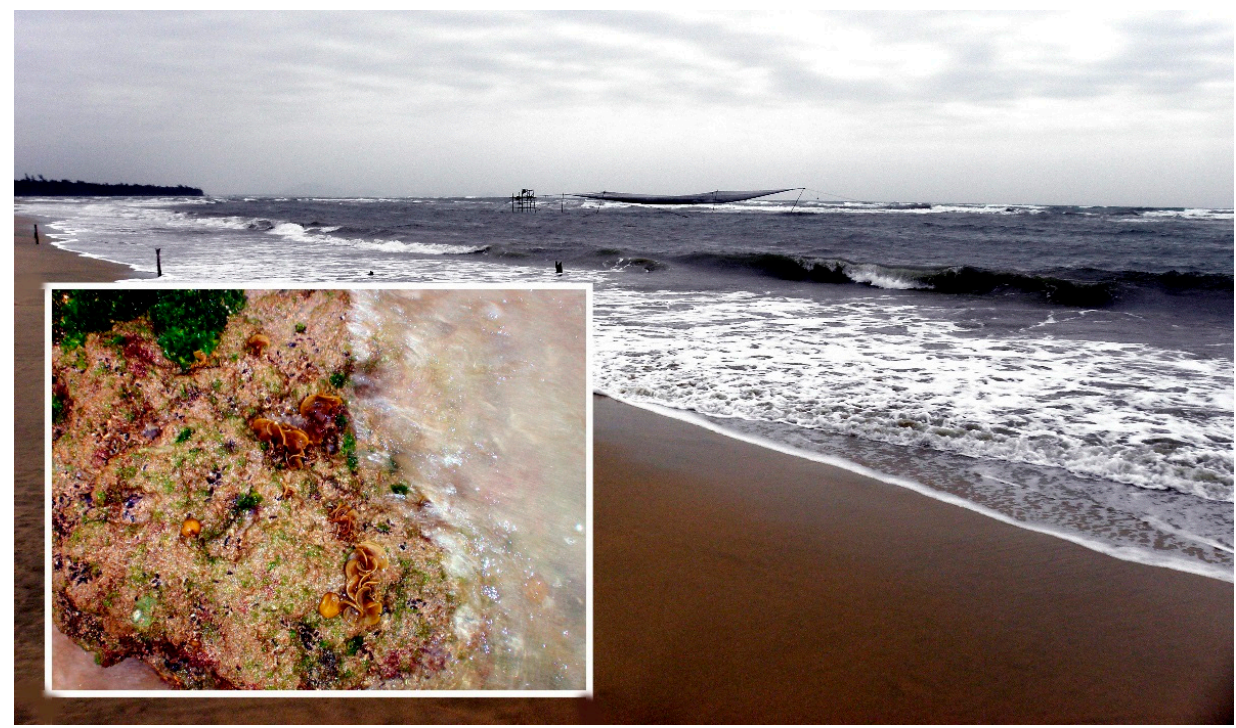

Figure 5. The vicinity of Wenchang City. Inset: Padina arborescens in association with turf algae in the middle intertidal zone, March 2012.

Meixia was characterized by an extensive stony intertidal area (cape site), above which was a sandy beach and a steeper upper intertidal zone, the coast being fringed by an offshore reef flat mostly composed of dead corals and, rarely, live coral colonies. Seaweeds were collected from this site at depths up to $4 \mathrm{~m}$. Here, we also observed in shallow water a bloom of the green alga Monostroma latissimum, which along the coastline formed extensive floating beds, many of which were washed ashore (Figure 6). 


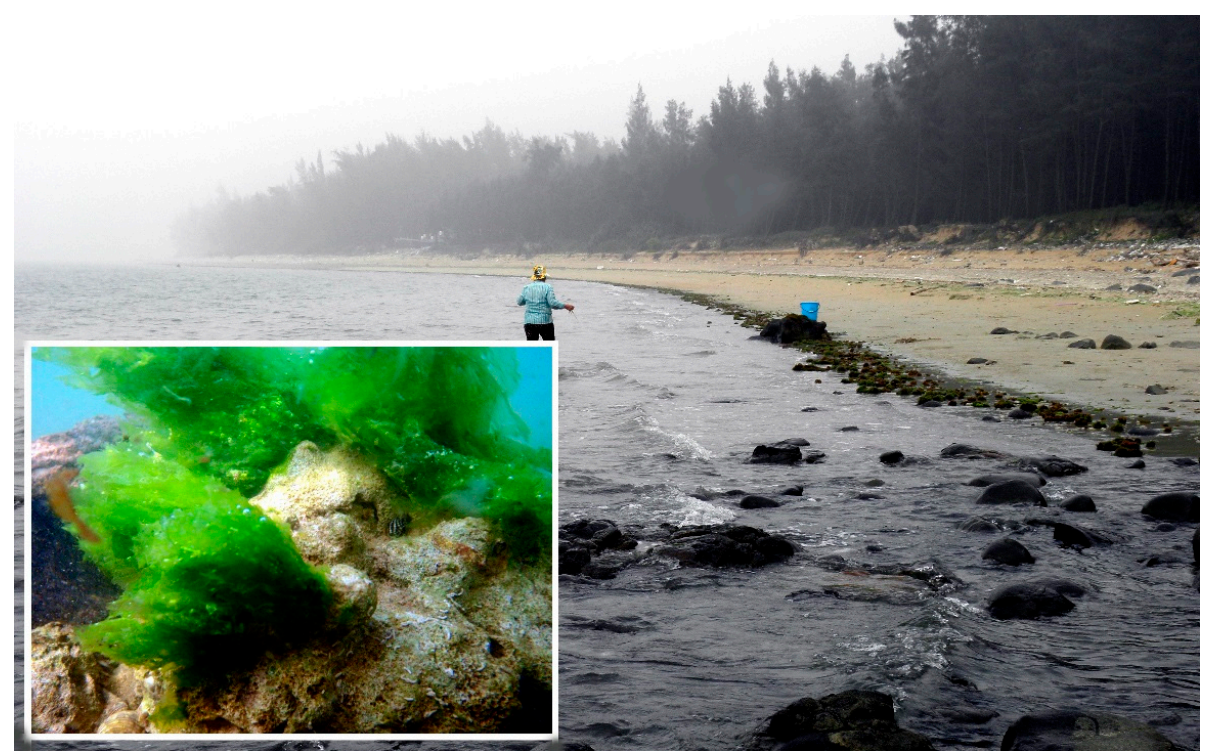

Figure 6. Meixia locality. Inset: Monostroma latissimum on dead coral block in the low intertidal zone, April 2012.

Ying Ge Hai was characterized by dead coral reef and seawater heavily polluted by coastal waste. Seaweeds were collected up to $4 \mathrm{~m}$ depth (Figure 7).

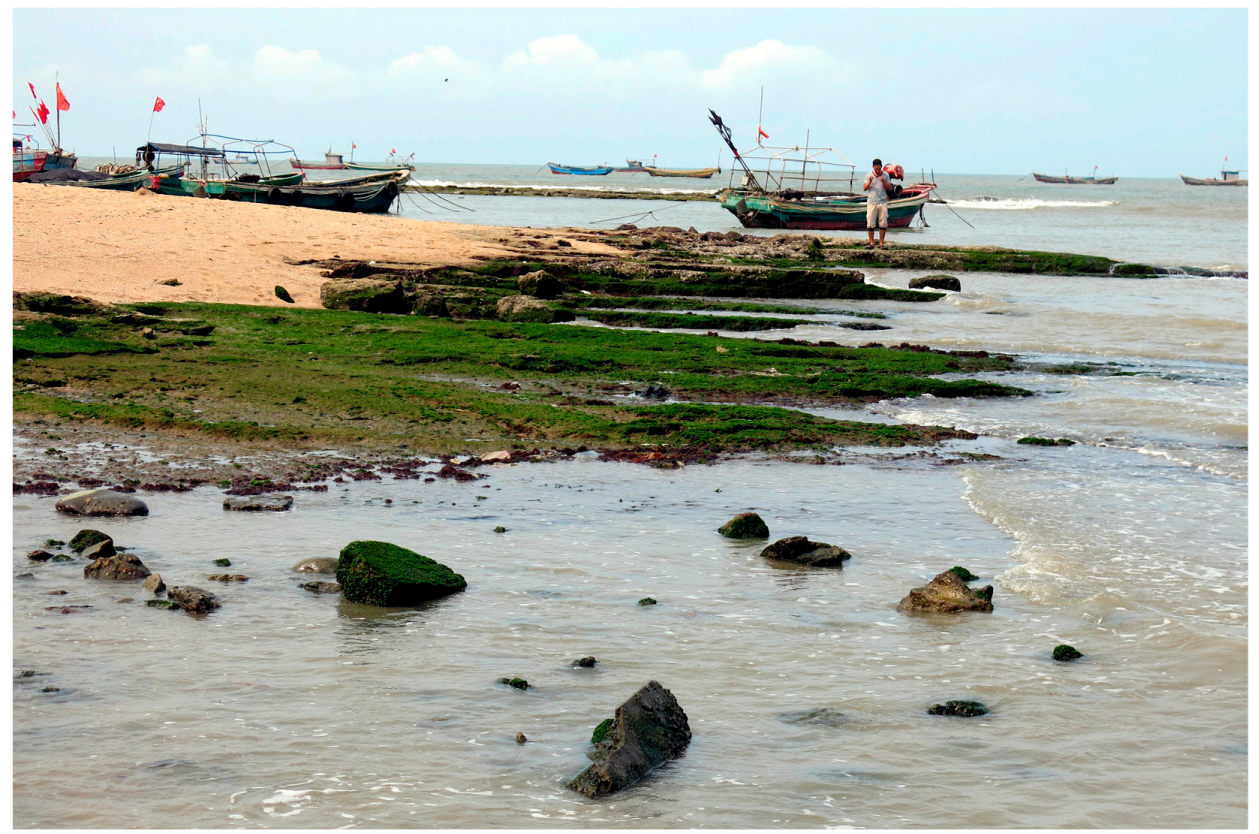

Figure 7. Ying Ge Hai, Ulva spp. belt, the middle intertidal zone, April 2014.

\subsection{Collection, Conservation, and Floristic Analysis of Samples}

During the German-Chinese expeditions $(1990,1992)$, sampling was carried out in the intertidal and shallow subtidal. Specimens were catalogued with consecutive numbers and were preserved as herbarium specimens. Duplicate sets of voucher specimens were prepared: one set is deposited at BRM in Bremerhaven, Germany; the other set in Qingdao, at the Institute of Oceanography, Chinese Academy of Sciences. Materials were identified by Xia and Bartsch in 1990-1994 and by Titlyanova and Titlyanov in 2007-2009. Anatomical studies were made using slides prepared from pre-soaked 
herbarium material and sectioned by hand with a razor blade. During the period from 2008 to 2016, macroalgae were collected in the upper, middle, and lower intertidal, and the upper subtidal (from 0.5 to $4 \mathrm{~m}$ depth during low tide) zones by Titlyanova and Titlyanov. In the upper subtidal zone, sampling of the marine plants was carried out via snorkeling and SCUBA diving (by Li) during both low- and hightides. Algae were collected from all types of substrata. The algal collection of 2008-2016 is deposited at the Zhirmunsky Institute of Marine Biology, Far Eastern Branch of the Russian Academy of Sciences.

Fresh and dried specimens were identified by Titlyanova and Titlyanov using monographic publications, floristic studies, and systematic articles cited in previous publications $[13,14,16,17]$. The systematics and nomenclature followed [21]. Hierarchical classification of the phylum Rhodophyta was assessed according to [22]. All publications concerning studies on Hainan Island have been reviewed in Titlyanova et al. in 2014. The previously known and newly recorded species for Hainan and China were verified using AlgaeBase, the Catalogue of Life China 2010: Annual Checklist and the Checklist of Marine Biota of China Seas [21,23,24]. Results of this study are presented in table form as Table 1 "An annotated list of new records of benthic red algae (Rhodophyta) for Hainan Island coast between 1990 and 2016."

\section{Results}

Floristic Analysis of New Findings of Red Algae in Hainan Island in 1990/1992 and during the Period 2008-2016

A total of 64 taxa of red algae found in 1990 and 1992 were newly recorded for Hainan Island, and included 15 new records for China. Of the newly recorded red algae for Hainan Island, more than $60 \%$ were thin-filamentous, finely branched epiphytes (Table 1).

Most of the newly reported red algae species (63\%) are common to other tropical and subtropical areas. Thirty-seven percent (37\%) of these are found only in the Indo-Pacific, $24 \%$ restricted to temperate latitudes, and only $6 \%$ shared with Arctic and Antarctic floras. Seventy-eight percent (78\%) of the species recorded for the first time from Hainan Island in 1990 and 1992 were found again in 2008-2016. In 1990 and 1992, the largest number of new findings were from Xiaodong Hai (43 species), followed by Shalao ( 25 species), Ximao Zhou Island ( 24 species), Luhuitou Peninsula (21 species), Yalong Wan (19 species), Dadong Hai (16 species), Tian Ya Hai Jiao (12 species), Nanmai (10 species), Qukou (9 species), Meixia (8 species), Linchang (6 species), and 1-3 species from other areas.

During the period from 2008 to 2016, 54 taxa were newly recorded for Hainan Island of which 20 were new to China. More than $40 \%$ of newly recorded taxa were epiphytic. Between 1992 and 2016, the marine flora of red algae on Hainan Island has been increased by $60 \%$, of which $40 \%$ of species inhabit the Indo-Pacific, 17\% otherwise occur in temperate latitudes and 3 species occur in the Antarctic. During this period, the greatest number of new finds for Hainan Island were recorded for the Luhuitou Peninsula (34 taxa), then for Ying Ge Hai (15 taxa), followed by Xian Hai (11 taxa), Yalong Wan (9 taxa), Xiaodong Hai (9 taxa), Meixia (5 taxa), and Wenchang (3 taxa). 
Table 1. Annotated list of new records of benthic red algae (Rhodophyta) recorded from the shores of Hainan Island between 1990 and 2016.

\begin{tabular}{|c|c|c|c|c|}
\hline List of Species, Varieties and Forms & Sr & Lf & Dg & Sampling Times and Locations of Finds \\
\hline \multicolumn{5}{|l|}{ DIVISION RHODOPHYTA } \\
\hline \multicolumn{5}{|l|}{ CLASS STYLONEMATOPHYCEAE } \\
\hline \multicolumn{5}{|l|}{ ORDER STYLONEMATALES } \\
\hline Bangiopsis dumontioides (P. Crouan \& H. Crouan) V. Krishnmurthy & p & Ep & $\mathrm{T}, \mathrm{S}$ & $(\mathbf{\vee}) \mathrm{Xh}$ \\
\hline Chroodactylon ornatum (C. Agardh) Basson & \& & Ep & $\mathrm{T}, \mathrm{S}, \mathrm{M}$ & (中) $\mathrm{Dh}, \mathrm{Lc}, \mathrm{Lh}, \mathrm{Ty}, \mathrm{Yw}, \mathrm{Xh} /(\mathbf{\vee}) \mathrm{Lh}, \mathrm{Xh}$ \\
\hline Stylonema alsidii (Zanardini) K.M. Drew & \& & Ep & $\mathrm{T}, \mathrm{S}, \mathrm{M}$ & (\$)Dh, Lc, Lh, Mx, Nm, Qk, Sl, Ty, Yw, Xh, Xz/(ฯ)Dh, Mx, Lh, Wc, Yg, Yw, Xh \\
\hline \multicolumn{5}{|l|}{ ORDER ERYTHROPELTALES } \\
\hline \multicolumn{5}{|l|}{ Family Erythrotrichiaceae } \\
\hline Erythrotrichia carnea (Dillwyn) J. Agardh & \& & Ep & $\mathrm{T}, \mathrm{S}, \mathrm{M}, \mathrm{Ar}, \mathrm{An}$ & (4) Dh, Lc, Lh, Nm, Qk, Sl, Ty, Yw, Xc, Xh, Xn, Xz/(⿶)Dh, Lh, Mx, Wc, Yg, Yw, Xh \\
\hline Erythrocladia irregularis Rosenvinge & \& & Ep & $\mathrm{T}, \mathrm{S}, \mathrm{M}, \mathrm{Ar}, \mathrm{An}$ & (\$) $\mathrm{Ty}, \mathrm{Xh} /(\mathbf{Y}) \mathrm{Lh}, \mathrm{Wc}, \mathrm{Yw}_{\mathrm{w}} \mathrm{Xn}$ \\
\hline Porphyrostromium japonicum (Tokida) Kikuchi & 24 & Ep & $\mathrm{T}, \mathrm{S},(\mathrm{I}-\mathrm{P})$ & कQk \\
\hline Sahlingia subintegra (Rosenvinge) Kornmann & \& & Ep & $\mathrm{T}, \mathrm{S}, \mathrm{M}$ & (中)Sl, Ty, Xc, Xh, /(४)Lh, Mx, Wc, Yg, Yw, Xn \\
\hline \multicolumn{5}{|l|}{ CLASS FLORIDEOPHYCEAE } \\
\hline \multicolumn{5}{|l|}{ ORDER HILDENBRANDIALES } \\
\hline Hildenbrandia rubra (Sommerfelt) Meneghini & 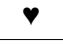 & $\mathrm{HS}, \mathrm{Cr}$ & $\mathrm{T}, \mathrm{S}, \mathrm{M}, \mathrm{Ar}, \mathrm{An}$ & (\)Dh, Lh, Mx, Xh \\
\hline \multicolumn{5}{|l|}{ ORDER NEMALIALES } \\
\hline \multicolumn{5}{|l|}{ Family Galaxauraceae } \\
\hline Dichotomaria falcata (Kjellman) Kurihara \& Masuda & mos & HS & $\mathrm{T}, \mathrm{S},(\mathrm{I}-\mathrm{P})$ & $(\mathbf{m}) X z$ \\
\hline Tricleocarpa cylindrica (J. Ellis \& Solander) Huisman \& Borowitzka & $\bullet$ & HS & $\mathrm{T}, \mathrm{S}$ & $(\mathbf{\vee}) \mathrm{Lh}, \mathrm{Xh}$ \\
\hline \multicolumn{5}{|l|}{ ORDER ACROCHAETIALES } \\
\hline \multicolumn{5}{|l|}{ Family Acrochaetiaceae } \\
\hline Acrochaetium catenulatum M. Howe & क\% & Ep & $\mathrm{T}, \mathrm{S}, \mathrm{M}$ & (\$)Xh \\
\hline Acrochaetium chaetomorphae (Tanaka \& Pham-Hoàng Hô) Heerebout & $\boldsymbol{\varphi}$ & Ep & $\mathrm{T}, \mathrm{S},(\mathrm{I}-\mathrm{P})$ & $(\boldsymbol{\vee}) \mathrm{Dh}, \mathrm{Lh}$ \\
\hline Acrochaetium secundatum (Lyngbye) Nägeli & \& & Ep & $\mathrm{T}, \mathrm{S}, \mathrm{M}$ & 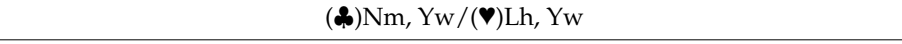 \\
\hline Acrochaetium subseriatum Børgesen & क\% & Ep & $\mathrm{T}, \mathrm{S},(\mathrm{I}-\mathrm{P})$ & (\$) $\mathrm{Xh}$ \\
\hline
\end{tabular}


Table 1. Cont.

\begin{tabular}{|c|c|c|c|c|}
\hline List of Species, Varieties and Forms & $\mathrm{Sr}$ & Lf & Dg & Sampling Times and Locations of Finds \\
\hline \multicolumn{5}{|l|}{ Family Colaconemataceae } \\
\hline Colaconema bonnemaisoniae Batters & 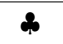 & Ep & $\mathrm{T}, \mathrm{S}, \mathrm{M}$ & (\$) $\mathrm{Xh}$ \\
\hline Colaconema daviesii (Dillwyn) Stegenga & 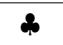 & Ep & $\mathrm{T}, \mathrm{S}, \mathrm{M}, \mathrm{Ar}, \mathrm{An}$ & $(\boldsymbol{q}) \mathrm{Xh} /(\mathbf{Y}) \mathrm{Lh}$ \\
\hline \multicolumn{5}{|l|}{ ORDER CORALLINALES } \\
\hline \multicolumn{5}{|l|}{ Family Corallinaceae } \\
\hline Amphiroa foliacea J.V. Lamouroux & \& & HS & $\mathrm{T}, \mathrm{S},(\mathrm{I}-\mathrm{P})$ & (\$)Lh, Sl/(ソ)Dh, Lh, Wc, Xh \\
\hline Hydrolithon boreale (Foslie) Y.M. Chamberlain & \& & Ep & $\mathrm{T}, \mathrm{S}, \mathrm{M}$ & $(\boldsymbol{\$}) \mathrm{Sl} /(\mathbf{\bullet}) \mathrm{Lh}, \mathrm{Yw}_{\mathrm{w}}$ \\
\hline Hydrolithon farinosum (J.V. Lamouroux) Penrose \& Y.M. Chamberlain & 4 & Ep & $\mathrm{T}, \mathrm{S}, \mathrm{M}$ & (\$) Dh, Lh, Qg, Qk, Sl, Ty, Yw Xh, Xz/(•)Lh, Wc, Yw, Xh \\
\hline \multirow{2}{*}{ Jania capillacea Harvey } & \multirow{2}{*}{4} & \multirow{2}{*}{ HS } & \multirow{2}{*}{$\mathrm{T}, \mathrm{S},(\mathrm{I}-\mathrm{P})$} & (\$)Dh, Lh, Mx, Nm, Sl, Ty, Yw, \\
\hline & & & & $\mathrm{Xh}, \mathrm{Xz}_{\mathrm{z}} / /(\mathbf{Y}) \mathrm{Lh}, \mathrm{Mx}, \mathrm{Yg}_{\mathrm{g}} \mathrm{Yw}_{\mathrm{w}} \mathrm{Xh}$ \\
\hline Jania longiarthra E.Y. Dawson & $\varphi$ & HS & $\mathrm{T}, \mathrm{S}$ & $(\bullet) X n$ \\
\hline Jania pumila J.V. Lamouroux & \& & HS & $\mathrm{T}, \mathrm{S}$ & 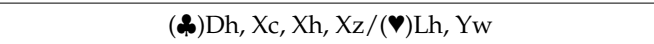 \\
\hline Jania ungulata f. brevior (Yendo) Yendo & \& & HS & $\mathrm{T}, \mathrm{S},(\mathrm{I}-\mathrm{P})$ & $(\mathbf{q}) \mathrm{Lh}, \mathrm{Xh} /(\mathbf{P}) \mathrm{Dh}, \mathrm{Wc}, \mathrm{Yw}, \mathrm{Xh}$ \\
\hline Lithophyllum tumidulum Foslie & $\mathbf{v}$ & $\mathrm{HS}, \mathrm{Cr}$ & $\mathrm{T}, \mathrm{S},(\mathrm{I}-\mathrm{P})$ & $(\mathbf{Y})$ Lh, Wc \\
\hline Neogoniolithon megalocystum (Foslie) & $\varphi$ & $\mathrm{HS}, \mathrm{Cr}$ & $\mathrm{T}, \mathrm{S}, \mathrm{M},(\mathrm{I}-\mathrm{P})$ & (४)Lh \\
\hline \multicolumn{5}{|l|}{ Setchell \& L.R. Mason } \\
\hline Pneophyllum confervicola (Kützing) Y.M. Chamberlain & 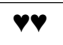 & $\mathrm{HS}, \mathrm{Cr}$ & $\mathrm{T}, \mathrm{S}, \mathrm{M}$ & ${ }^{*} \mathrm{Lh}, \mathrm{Wc}$ \\
\hline Pneophyllum fragile Kützing & 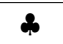 & $\mathrm{HS}, \mathrm{Cr}$ & $\mathrm{T}, \mathrm{S}, \mathrm{M}$ & (\$) Dh, Lh, Mx, Nm, Sl, Ty, Yw, Xh, Xz/(Ү)Lh, Mx, Wc, Yw \\
\hline \multicolumn{5}{|l|}{ Family Lithothamniaceae } \\
\hline Lithothamnion intermedium Kjellman & $\vee$ & $\mathrm{HS}, \mathrm{Cr}$ & $\mathrm{T}, \mathrm{S}, \mathrm{M}$ & $(\vee) Y_{w}$ \\
\hline Lithothamnion phymatodeum Foslie & $\varphi$ & $\mathrm{HS}, \mathrm{Cr}$ & $\mathrm{T}, \mathrm{S}, \mathrm{M},(\mathrm{I}-\mathrm{P})$ & $(\mathbf{\bullet}) \mathrm{Lh}$ \\
\hline \multicolumn{5}{|l|}{ ORDER CERAMIALES } \\
\hline \multicolumn{5}{|l|}{ Family Ceramiaceae } \\
\hline Antithamnion antillanum Børgesen & \& & Ep & $\mathrm{T}, \mathrm{S}$ & $(\boldsymbol{\uparrow}) \mathrm{Sl} /(\boldsymbol{\vee}) \mathrm{Dh}, \mathrm{Lh}$ \\
\hline Antithamnionella breviramosa (E.Y. Dawson) Wollaston & $\varphi$ & Fo & $\mathrm{T}, \mathrm{S}$ & $(\vee) \mathrm{Xh}$ \\
\hline Antithamnionella elegans (Berthold) J.H. Price \& D.M. John & $\vee$ & Ep & $\mathrm{T}, \mathrm{S}, \mathrm{M}$ & (Ү)Lh, Yw, Xn \\
\hline Antithamnionella spirographidis (Schiffner) E.M. Wollaston & \& & Ep & $\mathrm{T}, \mathrm{S}, \mathrm{M}$ & (\$)Sl \\
\hline Centroceras japonicum Itono & $\varphi$ & Ep & $\mathrm{T}, \mathrm{S},(\mathrm{I}-\mathrm{P})$ & $(\mathbf{\vee}) \mathrm{Lh}, \mathrm{Yw}_{\mathrm{w}}$ \\
\hline Centroceras minutum Yamada & \& & Ep & $\mathrm{T}, \mathrm{S},(\mathrm{I}-\mathrm{P})$ & (\$) $) \mathrm{Sl}, \mathrm{Xh}, \mathrm{Xz} /(\mathbf{Y}) \mathrm{Lh}$ \\
\hline
\end{tabular}


Table 1. Cont.

\begin{tabular}{|c|c|c|c|c|}
\hline List of Species, Varieties and Forms & $\mathrm{Sr}$ & Lf & Dg & Sampling Times and Locations of Finds \\
\hline Ceramium aduncum Nakamura & \& & Ep & $\mathrm{T}, \mathrm{S},(\mathrm{I}-\mathrm{P})$ & (\$) $\mathrm{Nm}, \mathrm{Xh} /(\mathbf{\vee}) \mathrm{Lh}$ \\
\hline Ceramium borneense Weber-van Bosse & $\$$ & Ep & $\mathrm{T}, \mathrm{S},(\mathrm{I}-\mathrm{P})$ & $(\mathbf{q}) \mathrm{Xh} /(\mathbf{v}) \mathrm{Lh}, \mathrm{Yw}, \mathrm{Xh}$ \\
\hline Ceramium camouii E.Y. Dawson & $\vee$ & Ep, HS & $\mathrm{T}, \mathrm{S},(\mathrm{I}-\mathrm{P})$ & $(\boldsymbol{Y}) \mathrm{Lh}, \mathrm{Xh}$ \\
\hline Ceramium cimbricum H.E. Petersen & \& & Ep & $\mathrm{T}, \mathrm{S}, \mathrm{M}$ & (\$) $\mathrm{Xh} /(\mathbf{Y}) \mathrm{Lh}, \mathrm{Wc}, \mathrm{Yg}, \mathrm{Xh}, \mathrm{Xn}$ \\
\hline Ceramium cingulatum Weber-van Bosse & ach & Ep & $\mathrm{T}, \mathrm{S}$ & (\$) $\mathrm{Dh}, \mathrm{Lc}, \mathrm{Lh}, \mathrm{Nm}, \mathrm{Sl}, \mathrm{Ty}, \mathrm{Yw}, \mathrm{Xh}, \mathrm{Xz}$, /(ヤ)Lh, Yg, Yw \\
\hline Ceramium codii (H. Richards) Mazoyer & $\varphi \varphi$ & Ep & $\mathrm{T}, \mathrm{S}$ & $(\mathbf{\Upsilon}) \operatorname{Lh}$ \\
\hline Ceramium comptum Børgesen & $\vee$ & Ep & $\mathrm{T}, \mathrm{S}$ & ${ }^{*} \mathrm{Lh}$ \\
\hline Ceramium macilentum J. Agardh & $\$$ & Ep & $\mathrm{T}, \mathrm{S},(\mathrm{I}-\mathrm{P})$ & (中)Sl, Xh, Xz/(•)Lh, Xh, Xn \\
\hline Ceramium marshallense E.Y. Dawson & 2क & Ep, HS & $\mathrm{T}, \mathrm{S},(\mathrm{I}-\mathrm{P})$ & (\$)Xh, Xz/(•)Lh, Yg, Yw, Xh \\
\hline Ceramium procumbens Setchell \& N.L. Gardner & $\varphi \varphi$ & Ep & $\mathrm{T}, \mathrm{S},(\mathrm{I}-\mathrm{P})$ & $(\boldsymbol{\vee}) \mathrm{Lh}, \mathrm{Xh}$ \\
\hline Ceramium serpens Setchell \& N.L. Gardner & $\varphi$ & Ep & $\mathrm{T}, \mathrm{S}$ & $(\uparrow) X n$ \\
\hline Ceramium tenerrimum (G. Martens) Okamura & \& & Ep & $\mathrm{T}, \mathrm{S}$ & \\
\hline Ceramium vagans P.C. Silva & \& & Ep & $\mathrm{T}, \mathrm{S}$ & \\
\hline Corallophila kleiwegii Weber-van Bosse & \& & Ep, HS & $\mathrm{T}, \mathrm{S},(\mathrm{I}-\mathrm{P})$ & \\
\hline Gayliella fimbriata (Setchell \& N.L. Gardner) T.O. Cho \& S.M. Boo & $\vee$ & Ep, & $\mathrm{T}, \mathrm{S},(\mathrm{I}-\mathrm{P})$ & \\
\hline Gayliella mazoyerae T.O. Cho, Fredericq \& Hommersand & $\$$ & Ep, HS & $\mathrm{T}, \mathrm{S}$ & \\
\hline Gayliella taylorii (E.Y. Dawson) T.O. Cho \& S.M. Boo & $\vee$ & Ep & $\mathrm{T}, \mathrm{S}$ & \\
\hline Aglaothamnion cordatum (Børgesen) Feldmann-Mazoyer & 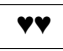 & Ep & $\mathrm{T}, \mathrm{S}$ & \\
\hline Crouania attenuata (C. Agardh) J. Agardh & \& & Ep & $\mathrm{T}, \mathrm{S}, \mathrm{M}$ & \\
\hline \multicolumn{5}{|l|}{ Family Delesseriaceae } \\
\hline Taenioma perpusillum (J. Agardh) J. Agardh & $\$$ & Ep, HS & $\mathrm{T}, \mathrm{S}$ & \\
\hline \multicolumn{5}{|l|}{ Family Rhodomelaceae } \\
\hline Acanthophora aokii Okamura & $\vee$ & HS & $\mathrm{T}, \mathrm{S},(\mathrm{I}-\mathrm{P})$ & \\
\hline Bryocladia cervicornis (Kützing) F. Schmitz & $\varphi \varphi$ & Ep & $\mathrm{T}, \mathrm{S},(\mathrm{I}-\mathrm{P})$ & \\
\hline Chondria dangeardii E.Y. Dawson & $\varphi \varphi$ & Ep & $\mathrm{T}, \mathrm{S}$ & \\
\hline Chondria minutula Weber-van Bosse & $\varphi \varphi$ & Ep & $\mathrm{T}, \mathrm{S},(\mathrm{I}-\mathrm{P})$ & \\
\hline Chondria pygmaea Garbary \& Vandermeulen & $\varphi \varphi$ & Ep & $\mathrm{T}, \mathrm{S}$ & \\
\hline Chondria repens Børgesen & \& & Ep & $\mathrm{T}, \mathrm{S},(\mathrm{I}-\mathrm{P})$ & \\
\hline
\end{tabular}


Table 1. Cont.

\begin{tabular}{|c|c|c|c|c|}
\hline List of Species, Varieties and Forms & $\mathrm{Sr}$ & Lf & Dg & Sampling Times and Locations of Finds \\
\hline Chondrophycus articulatus (C.K. Tseng) K.W. Nam & 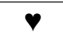 & HS & $\mathrm{T}, \mathrm{S},(\mathrm{I}-\mathrm{P})$ & \\
\hline Herposiphonia insidiosa (Greville ex J. Agardh) Falkenberg & $\varphi$ & HS & $\mathrm{T}, \mathrm{S},(\mathrm{I}-\mathrm{P})$ & \\
\hline Herposiphonia parca Setchell & \& & Ep & $\mathrm{T}, \mathrm{S},(\mathrm{I}-\mathrm{P})$ & \\
\hline Herposiphonia secunda (C. Agardh) Ambronn & \& & Ep & $\mathrm{T}, \mathrm{S}$ & \\
\hline Herposiphonia secunda f. tenella (C. Agardh) M.J. Wynne & ap & HS & $\mathrm{T}, \mathrm{S}$ & \\
\hline Laurencia decumbens Kützing & $\bullet$ & HS & $\mathrm{T}, \mathrm{S}$ & \\
\hline Laurencia pinnata Yamada & $\bullet$ & HS & $\mathrm{T}, \mathrm{S},(\mathrm{I}-\mathrm{P})$ & \\
\hline Laurencia silvae J.F. Zhang \& B.M. Xia & $\bullet$ & Ep & $\mathrm{T}, \mathrm{S},(\mathrm{I}-\mathrm{P})$ & \\
\hline Lophosiphonia cristata Falkenberg & $\varphi \varphi$ & Ep & T,S & \\
\hline Melanothamnus ferulaceus (Suhr ex J. Agardh) Díaz-Tapia \& Maggs & \& & Ep & $\mathrm{T}, \mathrm{S}$ & \\
\hline Melanothamnus pseudovillum (Hollenberg) Díaz-Tapia \& Maggs & 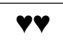 & Ep & $\mathrm{T}, \mathrm{S}$ & \\
\hline Melanothamnus savatieri (Hariot) Díaz-Tapia \& Maggs & YY & Ep & $\mathrm{T}, \mathrm{S}$ & \\
\hline Palisada concreta (A.B. Cribb) K.W. Nam & $\bullet$ & HS & $\mathrm{T}, \mathrm{S},(\mathrm{I}-\mathrm{P})$ & \\
\hline Palisada intermedia (Yamada) K.W. Nam & $\bullet$ & HS & $\mathrm{T}, \mathrm{S}$ & \\
\hline Palisada parvipapillata (C.K. Tseng) K.W. Nam & $\$$ & HS & $\mathrm{T}, \mathrm{S},(\mathrm{I}-\mathrm{P})$ & \\
\hline Palisada papillosa (C. Agardh) K.W. Nam & $\$$ & HS & $\mathrm{T}, \mathrm{S},(\mathrm{I}-\mathrm{P})$ & \\
\hline Pleonosporium borreri (Smith) Nägeli & 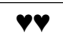 & Ep & $\mathrm{T}, \mathrm{S}, \mathrm{M}$ & \\
\hline Polysiphonia exilis Harvey & YY & Ep & $\mathrm{T}, \mathrm{S}$ & \\
\hline Polysiphonia scopulorum Harvey & \& & Ep & $\mathrm{T}, \mathrm{S}$ & \\
\hline Polysiphonia scopulorum var. villum (J. Agardh) Hollenberg & asp & Ep & $\mathrm{T}, \mathrm{S}$ & \\
\hline Polysiphonia subtilissima Montagne & \& & Ep & $\mathrm{T}, \mathrm{S}$ & \\
\hline Tolypiocladia condensata (Weber-van Bosse) P.C. Silva & aps & HS & $\mathrm{T}, \mathrm{S},(\mathrm{I}-\mathrm{P})$ & \\
\hline Tolypiocladia glomerulata (C. Agardh) F. Schmitz & \& & HS, Ep & $\mathrm{T}, \mathrm{S},(\mathrm{I}-\mathrm{P})$ & \\
\hline Vertebrata reptabunda (Suhr) Diaz-Tapia \& Maggs & $\&$ & Ep & $\mathrm{T}, \mathrm{S}$ & \\
\hline \multicolumn{5}{|l|}{ Family Wrangeliaceae } \\
\hline Gordoniella yonakuniensis (Yamada \& T. Tanaka) Itono & \& & Ep & $\mathrm{T}, \mathrm{S},(\mathrm{I}-\mathrm{P})$ & \\
\hline Wrangelia argus (Montagne) Montagne & \& & HS, Ep & $\mathrm{T}, \mathrm{S}$ & \\
\hline \multicolumn{5}{|l|}{ Family Dasyaceae } \\
\hline Heterosiphonia crispella (C. Agardh) M.J. Wynne & $\varphi \varphi$ & Ep & $\mathrm{T}, \mathrm{S}$ & \\
\hline
\end{tabular}


Table 1. Cont

\begin{tabular}{|c|c|c|c|c|}
\hline List of Species, Varieties and Forms & Sr & Lf & $\mathrm{Dg}$ & Sampling Times and Locations of Finds \\
\hline \multicolumn{5}{|l|}{ ORDER GELIDIALES } \\
\hline \multicolumn{5}{|l|}{ Family Gelidiaceae } \\
\hline Gelidium crinale (Hare ex Turner) Gaillon & \& & HS & $\mathrm{T}, \mathrm{S}, \mathrm{M}, \mathrm{An}$ & \\
\hline Gelidiophycus divaricatus (G. Martens) G.H. Boo, J.K. Park \& S.M. Boo & \& & HS & $\mathrm{T}, \mathrm{S},(\mathrm{I}-\mathrm{P})$ & (\$) $\mathrm{Mx}, \mathrm{Sl} /(\boldsymbol{\vee}) \mathrm{Lh}, \mathrm{Wc}$ \\
\hline \multicolumn{5}{|l|}{ Family Gelidiellaceae } \\
\hline Gelidiella lubrica (Kützing) Feldmann \& G. Hamel & क4 & Ep & $\mathrm{T}, \mathrm{S}$ & (中) $\mathrm{Xh}$ \\
\hline Parviphycus adnatus (E.Y. Dawson) B. Santelices & 24 & Ep & $\mathrm{T}, \mathrm{S}$ & (中) $\mathrm{Lh}, \mathrm{Sl}, \mathrm{Xh} /(\mathbf{(}) \mathrm{Lh}$ \\
\hline Parviphycus pannosus (Feldmann) G. Furnari & क4 & Ep & $\mathrm{T}, \mathrm{S}$ & \&Lh, Xh, Xz/*Dh, Lh, Wc, Yw, Xh \\
\hline \multicolumn{5}{|l|}{ Family Pterocladiaceae } \\
\hline Pterocladiella capillacea (S.G. Gmelin) Santelices \& Hommersand & 4 & HS & $\mathrm{T}, \mathrm{S}, \mathrm{M}$ & $(\boldsymbol{中}) \mathrm{Xh} /(\mathbf{\bullet}) \mathrm{Lh}, \mathrm{Xn}$ \\
\hline \multicolumn{5}{|l|}{ ORDER GIGARTINALES } \\
\hline \multicolumn{5}{|l|}{ Family Cystocloniaceae } \\
\hline Hypnea cenomyce J. Agardh & 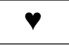 & HS & $\mathrm{T}, \mathrm{S}$ & (४)Lh, Mx, Yw \\
\hline Hypnea chordacea Kützing & 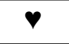 & HS & $\mathrm{T}, \mathrm{S},(\mathrm{I}-\mathrm{P})$ & (४) $\mathrm{Yg}$ \\
\hline Hypnea esperi Bory & $\varphi$ & HS & T,S,M,An & $(\bullet) \operatorname{Lh}, Y_{w}$ \\
\hline Hypnea musciformis var. esperi J. Agardh & \& & Ep & $\mathrm{T}, \mathrm{S},(\mathrm{I}-\mathrm{P})$ & (\$) Dh, Lc, Nm, Xh \\
\hline Hypnea nidulans Setchell & $\vee$ & HS & $\mathrm{T}, \mathrm{S},(\mathrm{I}-\mathrm{P})$ & $(\mathbf{\vee}) \operatorname{Lh}$ \\
\hline Hypnea valentiae (Turner) Montagne & $\varphi$ & HS & $\mathrm{T}, \mathrm{S}$ & (४)Lh, Mx, Yg, Xh, Xn \\
\hline \multicolumn{5}{|l|}{ Family Gigartinaceae } \\
\hline Chondracanthus intermedius (Suringar) Hommersand & $\vee$ & $\mathrm{HS}, \mathrm{Cr}$ & $\mathrm{T}, \mathrm{S},(\mathrm{I}-\mathrm{P})$ & $(\bullet) M x, X n$ \\
\hline Chondracanthus tenellus (Harvey) Hommersand & 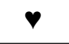 & HS & $\mathrm{T}, \mathrm{S},(\mathrm{I}-\mathrm{P})$ & $(\vee) X n$ \\
\hline \multicolumn{5}{|l|}{ Family Rhizophyllidaceae } \\
\hline Portieria hornemannii (Lyngbye) P.C. Silva & \& & HS & $\mathrm{T}, \mathrm{S},(\mathrm{I}-\mathrm{P})$ & (\$)Sl \\
\hline \multicolumn{5}{|l|}{ ORDER PEYSSONNELIALES } \\
\hline \multicolumn{5}{|l|}{ Family Peyssonneliaceae } \\
\hline Peyssonnelia boergesenii Weber-van Bosse & $\varphi$ & $\mathrm{HS}, \mathrm{Cr}$ & $\mathrm{T}, \mathrm{S}$ & $(\bullet) \mathrm{Lh}, \mathrm{Yw}$ \\
\hline Peyssonnelia inamoena Pilger & $\varphi$ & $\mathrm{HS}, \mathrm{Cr}$ & $\mathrm{T}, \mathrm{S}$ & $(\vee) \operatorname{Lh}, Y_{w}$ \\
\hline Peyssonnelia rubra (Greville) J. Agardh & क4 & $\mathrm{HS}, \mathrm{Cr}$ & $\mathrm{T}, \mathrm{S}$ & 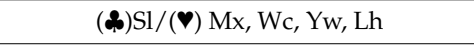 \\
\hline \multicolumn{5}{|l|}{ ORDER GRACILARIALES } \\
\hline Family Gracilariaceae & & & & \\
\hline
\end{tabular}


Table 1. Cont

\begin{tabular}{|c|c|c|c|c|}
\hline List of Species, Varieties and Forms & $\mathrm{Sr}$ & Lf & $\mathrm{Dg}$ & Sampling Times and Locations of Finds \\
\hline $\begin{array}{c}\text { Gracilaria changii (B.M. Xia \& I.A. Abbott) I.A. Abbott, } \\
\text { J. Zhang \& B.M. Xia }\end{array}$ & 2 & HS & T,S,(I-P) & (\$) $\mathrm{Nm}$ \\
\hline Gracilaria coronopifolia J. Agardh & 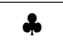 & HS & $\mathrm{T}, \mathrm{S},(\mathrm{I}-\mathrm{P})$ & $(\mathbf{m}) \mathrm{Xn} /(\mathbf{P}) \mathrm{Lh}, \mathrm{Xn}$ \\
\hline Gracilaria textorii (Suringar) De Toni & $\varphi$ & HS & $\mathrm{T}, \mathrm{S}$ & $(\boldsymbol{\vee}) \mathrm{Xn}$ \\
\hline \multicolumn{5}{|l|}{ ORDER HALYMENIALES } \\
\hline \multicolumn{5}{|l|}{ Family Halymeniaceae } \\
\hline Grateloupia asiatica S. Kawaguchi \& H.W. Wang & $\vee$ & HS & $\mathrm{T}, \mathrm{S}$ & $(\boldsymbol{\vee}) \mathrm{Yg}, \mathrm{Xn}$ \\
\hline Grateloupia carnosa Yamada \& Segawa & $\vee$ & HS & $\mathrm{T}, \mathrm{S},(\mathrm{I}-\mathrm{P})$ & $(\bullet) Y g$ \\
\hline Grateloupia filicina (J.V. Lamouroux) C. Agardh & $\varphi$ & HS & $\mathrm{T}, \mathrm{S}, \mathrm{M}, \mathrm{An}$ & $(\mathbf{Y}) \mathrm{Lh}, \mathrm{Yg}, \mathrm{Xn}$ \\
\hline \multicolumn{5}{|l|}{ ORDER RHODYMENIALES } \\
\hline \multicolumn{5}{|l|}{ Family Champiaceae } \\
\hline Champia parvula (C. Agardh) Harvey & \& & HS & $\mathrm{T}, \mathrm{S}, \mathrm{M}$ & (中) $\mathrm{Yw}_{\mathrm{w}} \mathrm{Xh}, \mathrm{Xz} /(\boldsymbol{\varphi}) \mathrm{Dh}, \mathrm{Lh}, \mathrm{Yw}, \mathrm{Xh}$ \\
\hline Champia vieillardii Kützing & 240 & HS & $\mathrm{T}, \mathrm{S}$ & (\$)Lh/(ソ)Dh, Lh, Xh \\
\hline \multicolumn{5}{|l|}{ Family Lomentariaceae } \\
\hline Ceratodictyon scoparium (Montagne \& Millardet) R.E. Norris & $\varphi \varphi$ & HS & $\mathrm{T}, \mathrm{S}$ & (ү)Dh, Lh, Wc, Xh \\
\hline Lomentaria corallicola Børgesen & 240 & HS & $\mathrm{T}, \mathrm{S}$ & $(\mathbf{h}) \mathrm{Sl}, \mathrm{Xh} /(\boldsymbol{\bullet}) \mathrm{Lh}, \mathrm{Dh}$ \\
\hline \multicolumn{5}{|l|}{ Family Hymenocladiaceae } \\
\hline $\begin{array}{l}\text { Asteromenia anastomosans (Weber-van Bosse) G.W. Saunders, C.E. } \\
\text { Lane, C.W. Schneider \& Kraft }\end{array}$ & 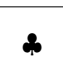 & HS & $\mathrm{T}, \mathrm{S}$ & (中) $Q g$ \\
\hline
\end{tabular}

Column 1 lists the new algal records for Hainan Island and for China obtained during the Chinese-German expeditions in 1990 and 1992 and during the more recent investigations undertaken from 2008 to 2016. Column 2 (Sr) indicates the status of the species found: In 1990 and 1992, $\mathbf{\phi}=$ new records for Hainan Island; $\boldsymbol{中} \boldsymbol{中}=$ new records for China. In 2008-2016: $\boldsymbol{Y}=$ new records for Hainan Island; $\boldsymbol{\vee}=$ new records for China. Column 3 (Lf) indicates the life forms of the different taxa: Ep = epiphyte, HS = algae growing on hard substrate (epilithic), $\mathbf{C r}=$ crust form. Column 4 (Dg) shows the known distribution of the species across the various biogeographical zones: $\mathbf{T}=$ tropical; $\mathbf{S}=$ subtropical; $\mathbf{M}=$ temperate; $\mathbf{A n}=\mathrm{Antarctic}$ $\mathrm{Ar}=$ Arctic; TS $\mathbf{M}, \mathrm{An}=0 \mathrm{c}$ Ar = Aric; (IP) = A ( $\mathbf{L j}=$ Lingao Jiao; $\mathbf{L h}=$ Luhuitou; Nm = Nanmai; Mx = Meixia; $\mathbf{Q g}=$ Qinglan Gang; $\mathbf{Q k}=$ Qukou; $\mathbf{S} \mathbf{~ = ~ S h a l a o ; ~ T y ~ = ~ T i a n ~ Y a ~ H a i ~ J i a o ; ~ W c ~ = ~ W e n c h a n g ; ~} \mathbf{Y w}=$ Yalong Wan; $\mathbf{Y g}=$ Ying Ge Hai $\mathbf{X n}=$ Xian Hai; $\mathbf{X h}=$ Xiaodong Hai; $\mathbf{X} \mathbf{c}=$ Xincun; $\mathbf{X z}=$ Ximao Zhou 


\section{Discussion}

The records of algal species contained in our previous studies [13-20] suggest that conspicuous changes in the composition and diversity of red algae have occurred between the 1930s and 2012 . Counter-intuitively, there has been a decline in total species richness despite the appearance of many newly reported species. The floristic similarity in marine flora of Hainan Island between the two periods, 1930s-1970s and 2008-2012 [17], amounted to 33\%. By comparison the floristic similarity in marine flora between the two periods 1990/1992 and 2008-2012 was 65\%, i.e., the dissimilarity in the marine flora of Hainan Island between the 1930s and 1990s was two times that between the 1990s and 2008-2012.

At the same time, inspection of the species listed in Table 1 indicates that the enrichment (more than $40 \%$ ) of the red algal flora of Hainan during both periods occurred at the expense of finely branched, thin-filamentous epiphytes.

We believe that these decadal changes in the flora of the island of Hainan reflects the influence of both local anthropogenic impacts and of global change, including the following factors:

(1) The over-exploitation of the reef ecosystems, especially of algal grazing species of fish and invertebrates, developing from the 1950s-1970s [19].

(2) The eutrophication of shallow waters (bays) in the 1990s-2000s, occurring as a result most obviously of sewage disposal associated with coastal urbanization linked to the increase in tourism, but also with the growth in agriculture and mariculture [13]. This conclusion is supported by the fact that the largest numbers of new finds of red algae in 2008-2016 were from the most polluted areas of the coast, from such localities as Sanya Bay and Ying Ge Hai.

(3) The consequences of wider climate and environmental change, in particular the mass mortality of corals after bleaching such as occurred in the South China Sea in the summer of 1998 [17].

Acknowledgments: E.A. Titlyanov and T.V. Titlyanova are grateful to Christian Wiencke and Inka Bartsch for an invitation to work at the Alfred-Wegener-Institute for Polar and Marine Research and are also grateful to collaborators in the Botanical Department for technical help, use of facilities, and hospitality. Special thanks to Huang Hui for the invitation to work at the Marine Biological Station (South China Sea Institute of Oceanology, Chinese Academy of Sciences). This study was partially supported by the Russian Foundation for Basic Research (grant numbers 08-04-00735-a, 12-04-01424-a), by three research grants of the Alfred-Wegener Institute of Polar and Marine Research to T. Titlyanova and E. Titlyanov, and by the grant "China-Russia special funds 2009" from the Chinese Academy of Sciences.

Author Contributions: E.A. Titlyanov and T.V. Titlyanova conceived and designed the experiments; Inka Bartsch and Xia Bangmei performed the experiments (sampled marine plants in 1990 and 1992); T.V. Titlyanova, E.A. Titlyanov, X. Li collected marine plants in 2008-2016; Inka Bartsch and Xia Bangmei analyzed the materials sampled in 1990 and 1992; T.V. Titlyanova and E.A. Titlyanov analyzed the materials collected in 1990/1992 and 2008-2016; Titlyanova and E.A. Titlyanov wrote the paper.

Conflicts of Interest: The founding sponsors had no role in the design of the study; in the collection, analyses, or interpretation of data; in the writing of the manuscript, and in the decision to publish the results.

\section{References}

1. Sun, D.; Gagan, M.K.; Cheng, H.; Scott-Gagan, H.; Dykoski, C.A.; Edwards, R.L.; Su, R. Seasonal and interannual variability of the Mid-Holocene East Asian monsoon in coral $\delta^{18} \mathrm{O}$ records from the South China Sea. Earth Planet. Sci. Let. 2005, 237, 69-84. [CrossRef]

2. Levitus, S.; Boyer, T. World Ocean Atlas 1994, vol. 4, Temperature, NOAA Atlas NESDIS 4; U.S. Government Printing Office: Washington, DC, USA, 1994; p. 117.

3. Zhang, Q.; Xu, X.Z.; Long, X.M. A numerical study on internal tides in the northeast of the South China Sea. J. Trop. Oceanol. 1996, 14, 15-23. (In Chinese).

4. Zhang, Q.; Shi, Q.; Chen, G.; Fong, T.C.; Wong, D.C.; Huang, H.; Wang, H.; Zhao, M. Status monitoring and health assessment of Luhuitou fringing reef of Sanya, Hainan, China. Chin. Sci. Bull. 2006, 51, 81-88. [CrossRef]

5. Gurianova, E.F. Marine zoological expedition to Hainan Island. Vestnik AN SSSR 1959, 3, 89-92. (In Russian) 
6. Fiege, D.; Neumann, V.; Jinhe, L. Observations on coral reefs of Hainan Island, South China Sea. Mar. Pollut. Bull. 1994, 29, 84-89. [CrossRef]

7. Hodgson, G.; Yau, E.P.M. Physical and biological controls of coral communities in Hong Kong. Proceedings of 8th International Coral Reef Symposium, Balboa, Panama, 24-29 June 1996; Smithsonian Tropical Research Institute: Gamnoa, Panama, 1997.; pp. 459-464.

8. Huang, L.M.; Tan, Y.H.; Song, X.Y.; Huang, X.P.; Wang, H.K.; Zhang, S.; Dong, J.D.; Chen, R.Y. The status of the ecological environment and a proposed protection strategy in Sanya Bay, Hainan Island, China. Mar. Pollut. Bull. 2003, 47, 180-186. [CrossRef]

9. Zhang, G.; Que, H.; Liu, X.; Xu, H. Abalone mariculture in China. J. Shellfish Res. 2004, 23, 947-950.

10. Tadashi, K.; Dai, C.F.; Park, H.S.; Huang, H.; Ang, P.O. Status of coral reefs in East and North Asia (China, Hong Kong, Taiwan, South Korea and Japan). In Status of coral reefs of the world: 2008; Wilkinson, C., Ed.; Global Coral Reef Monitoring Network and Reef and Rainforest Research Centre: Townsville, Australia, 2008; pp. 145-158.

11. Zhao, M.X.; Yu, K.F.; Zhang, Q.M.; Shi, Q. Spatial pattern of coral diversity in Luhuitou fringing reef, Sanya, China. Acta Ecol. Sin. 2008, 28, 1419-1428.

12. Tseng, C.K. The past, present and future of phycology in China. Hydrobiologia 2004, 512, 11-20. [CrossRef]

13. Titlyanov, E.A.; Titlyanova, T.V.; Xia, B.M.; Bartsch, I. Checklist of marine benthic green algae (Chlorophyta) on Hainan, a subtropical island off the coast of China: comparisons between the 1930s and 1990-2009 reveal environmental changes. Bot. Mar. 2011, 54, 523-535. [CrossRef]

14. Titlyanov, E.A.; Titlyanova, T.V.; Huang, H.; Li, X.B. Seasonal changes in benthic algal communities of the upper subtidal zone in Sanya Bay (Hainan Island, China). J. Mar. Biol. Assoc. UK 2014, 91, 51-64. [CrossRef]

15. Titlyanov, E.A.; Titlyanova, T.V.; Li, X.B.; Hansen, G.; Huang, H. Seasonal changes in the intertidal algal communities of Sanya Bay (Hainan Island, China). J. Mar. Biol. Assoc. UK 2014, 94, 879-893. [CrossRef]

16. Titlyanova, T.; Titlyanov, E.; Xia, B.; Bartsch, I. New records of benthic marine green algae on Hainan Island, China. Nova Hedwigia 2012, 94, 441-470. [CrossRef] [PubMed]

17. Titlyanova, T.V.; Titlyanov, E.A.; Kalita, T.L. Marine algal flora of Hainan Island: a comprehensive synthesis. Coastal Ecosystems 2014, 1, 28-53.

18. Titlyanov, E.A.; Titlyanova, T.V.; Xia, B.M.; Bartsch, I. Retrospective analysis of diversity and species composition of marine macroalgae of hainan island (China). Ocean Sci. J. 2016, 51, 1-22. [CrossRef]

19. Titlyanov, E.A.; Titlyanova, T.V.; Belous, O.S.; Kalita, T.L. Inventory change (1990s-2010s) in the marine flora of Sanya Bay (Hainan Island, China). J. Mar. Biol. Assoc. UK 2015, 95, 461-470. [CrossRef]

20. Titlyanov, E.A.; Titlyanova, T.V.; Li, X.B.; Kalita, T.L.; Huang, H. Recent (2008-2012) seaweed flora of Hainan Island, South China Sea. Mar. Biol. Res. 2015, 11, 540-550. [CrossRef]

21. Guiry, M.D.; Guiry, G.M. World-wide electronic publication, National University of Ireland, Galway. Available online: http:/ / www.algaebase.org/ (accessed on 22 May 2017).

22. Saunders, G.W.; Hommersand, M.H. Assessing red algal supraordinal diversity and taxonomy in the context of contemporary systematic data. Am. J. Bot. 2004, 91, 1494-1507. [CrossRef] [PubMed]

23. Catalogue of Life: 2010 Annual Checklist. Available online: http://www.catalogueoflife.org/annualchecklist/2010 (accessed on 15 February 2017).

24. Liu, R. Checklist of marine biota of China Seas; China Scientific Book Service Co Ltd: Beijing, China, 2008; p. 1267.

(C) 2017 by the authors. Licensee MDPI, Basel, Switzerland. This article is an open access article distributed under the terms and conditions of the Creative Commons Attribution (CC BY) license (http://creativecommons.org/licenses/by/4.0/). 